\title{
Vessel Wall Contrast Enhancement on Magnetic Resonance Imaging May Be Suggestive for Future Development of Further Arterial Changes
}

\author{
Guillaume Saliou, Marc Tardieu, Marie Theaudin, Sarah Power, Kumaran Deiva
}

Keywords: Vessel wall enhancement, MRI, Infant, Vasculitis, Outcome

doi:10.1017/cjn.2016.251

Can J Neurol Sci. 2016; 43: 728-730

\section{INTRODUCTION}

We present a case of a 13-year-old boy who presented with subarachnoid haemorrhage due to a ruptured left carotid aneurysm, with underlying presumed CNS vasculitis. Initial angiography showed several fusiform and saccular aneurysms and stenosis in the anterior circulation, with posterior circulation sparing. Both the anterior and posterior circulation, however, did demonstrate concentric wall enhancement on MRI performed at that time. Diffuse and concentric vessel wall enhancement on MRI has been previously reported in patients with cerebral vasculitis. A presumptive diagnosis of vasculitis was made. Despite thorough investigation, the underlying aetiology of the vasculitis could not be determined, Treatment with steroids and immunosuppressant therapy was initiated, with little success. Follow-up MRI and four-year angiogram showed posterior circulation involvement in a similar pattern to the anterior circulation, with development of arterial stenosis and aneurysm. We hypothesize that wall enhancement could be an early predictor for further change in arteries that initially appear to be normal on angiography. To our knowledge, that finding has not been described previously.

\section{HiSTORY}

MRI of the intracranial arterial wall was also performed at the same time. Sequences included axial 2-mm thick T1SE-weighted sequences before and after gadolinium contrast injection. On the wall imaging study, both the anterior and posterior circulation demonstrated concentric arterial wall enhancement and thickening (Figure 1). These features involved internal carotid terminations to the M2 and A2 segments in the anterior circulation and the 2/3 distal basilar artery to the $\mathrm{P} 2$ segments.

\section{DISCUSSION}

\section{Case Report and Technique}

A 13-year-old boy presented with subarachnoid haemorrhage (SAH) due to a ruptured left carotid aneurysm treated with endovascular embolization, with underlying presumed CNS vasculitis. This was diagnosed six years previously, following a stroke in the right superficial sylvian territory with left-sided palsy, after which the patient was treated with aspirin. Following rupture of his aneurysm, the patient experienced two episodes of TIA (recurrence of left-sided palsy) within three months, and the treatment was switched to low-molecular-weight heparin at a therapeutic dose (tinzaparin), despite the presence of multiple cerebral aneurysms in the anterior circulation. Although the relationship with management changes was uncertain, no further strokes occurred following instigation of anticoagulation. Diagnosis of presumed CNS vasculitis was made in the context of a familial history of autoimmune disease (multiple sclerosis in an aunt and lupus in another aunt) and the presence of persistent anti-phospholipid antibodies (28 to a maximum of $51 \mathrm{U}$ of $\mathrm{IgG}$ ). All other inflammatory markers (ESR, native DNA, antib2GPI) were normal, as well as cerebrospinal fluid analysis. At the time of the $\mathrm{SAH}$, angiography showed several aneurysms and a stenosis in the anterior circulation, with posterior circulation sparing (Figure 1).

Steroid (prednisone $8 \mathrm{mg} /$ day, 3 years of treatment) and immunosuppressant therapy (cyclophosphamide $750 \mathrm{mg} / \mathrm{m}^{2}, 13$ sessions of treatment; rituximab $375 \mathrm{mg} / \mathrm{m}^{2}, 1$ dose) were initiated, without change of vessel wall enhancement on repeated yearly MRI during this time period, even though no new ischaemia-related symptoms occurred. Four years later, control angiography showed posterior circulation involvement, in a similar pattern to the anterior circulation, with development of a saccular basilar tip aneurysm and arterial stenosis (Figure 2). Beginning of dilatation of the basilar tip was retrospectively noted after reviewing the previous MRI. Because of the perceived high risk of ischaemic stroke, burr-hole treatment was performed to improve brain perfusion in case of further progression of the posterior circulation stenosis. Although risk of aneurysmal haemorrhage was still present, treatment of the remaining aneurysms was not undertaken, as procedural risks (either endovascular or surgical) were considered to be too high.

The main finding in this case is that vessel wall enhancement preceded the development of arterial aneurysmal dilatation and stenosis, with resultant risk of haemorrhage or ischaemic stroke. Indeed, the patient had severe stenosis of both internal carotid arteries, and the brain was mainly supplied by the vertebrobasilar

From le Service de Neuroradiologie, Hôpital Bicêtre, Le Kremlin-Bicêtre, France (GS, MT, MT); the Division of Neuroradiology, Toronto Western Hospital, Toronto, Ontario, Canada (SP); le Service de Neurologie Pédiatrique, Hôpital Bicêtre, Le Kremlin-Bicêtre, France (KD).

Received April 27, 2015. Final Revisions Submitted December 8, 2015. Date of ACCEPTANCE MARCH 11, 2016.

Correspondence to: Guillaume Saliou, Service de Neuroradiologie, Hôpital Bicêtre, 78 rue du General Leclerc, 94275 Le Kremlin-Bicêtre, France. E-mail: guillaume.saliou @bct.aphp.fr. 

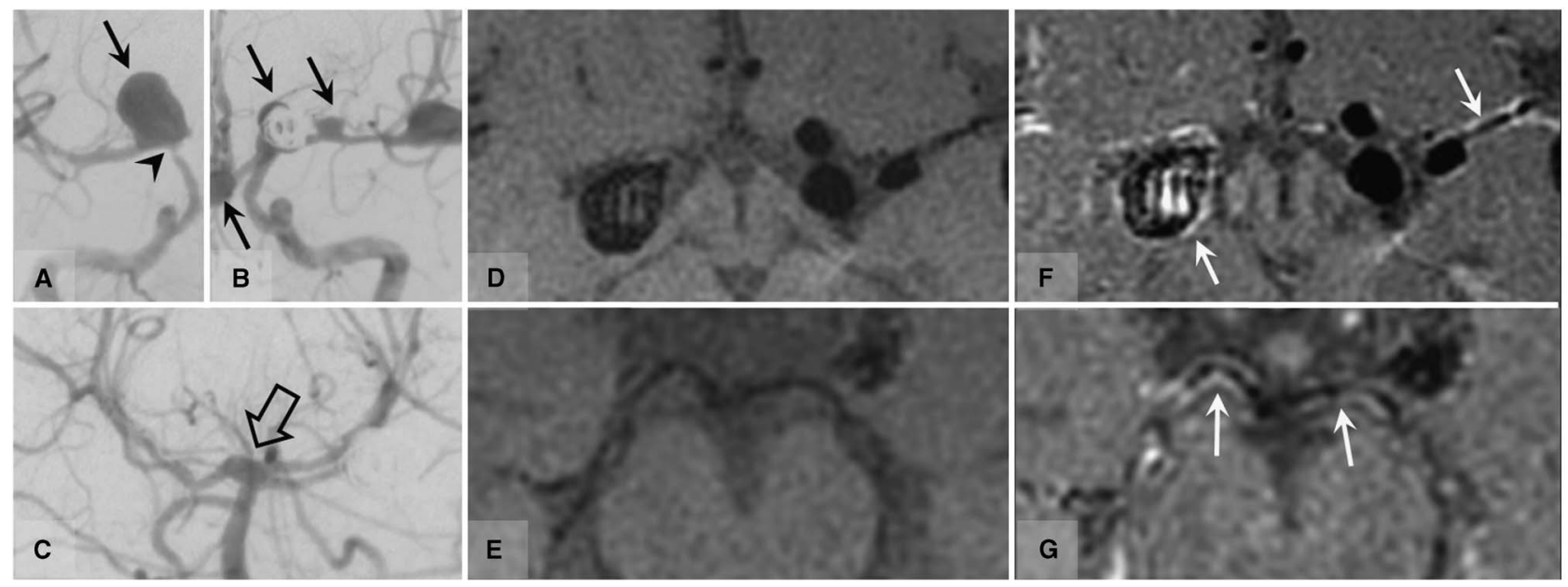

Figure 1: Angiography in frontal view performed April 2007 (A: right internal carotid; B: left internal carotid; C: basilar trunk) showed the aneurysms (A\&B: arrows) and a stenosis (A: arrowhead) in the carotid circulation while the basilar tip appeared normal (C: empty arrow). 1.5T MRI performed April 2007. Axial T1SE-weighted sequences performed before $(D \& E)$ and after $(F \& G)$ gadolinium contrast injection. Diffuse arterial wall enhancement is seen in the anterior (F: arrows) and posterior (G: arrows) circulation. Several aneurysms are identified in the carotid circulation $(D \& F)$. No posterior circulation aneurysm is present $(E \& G)$.
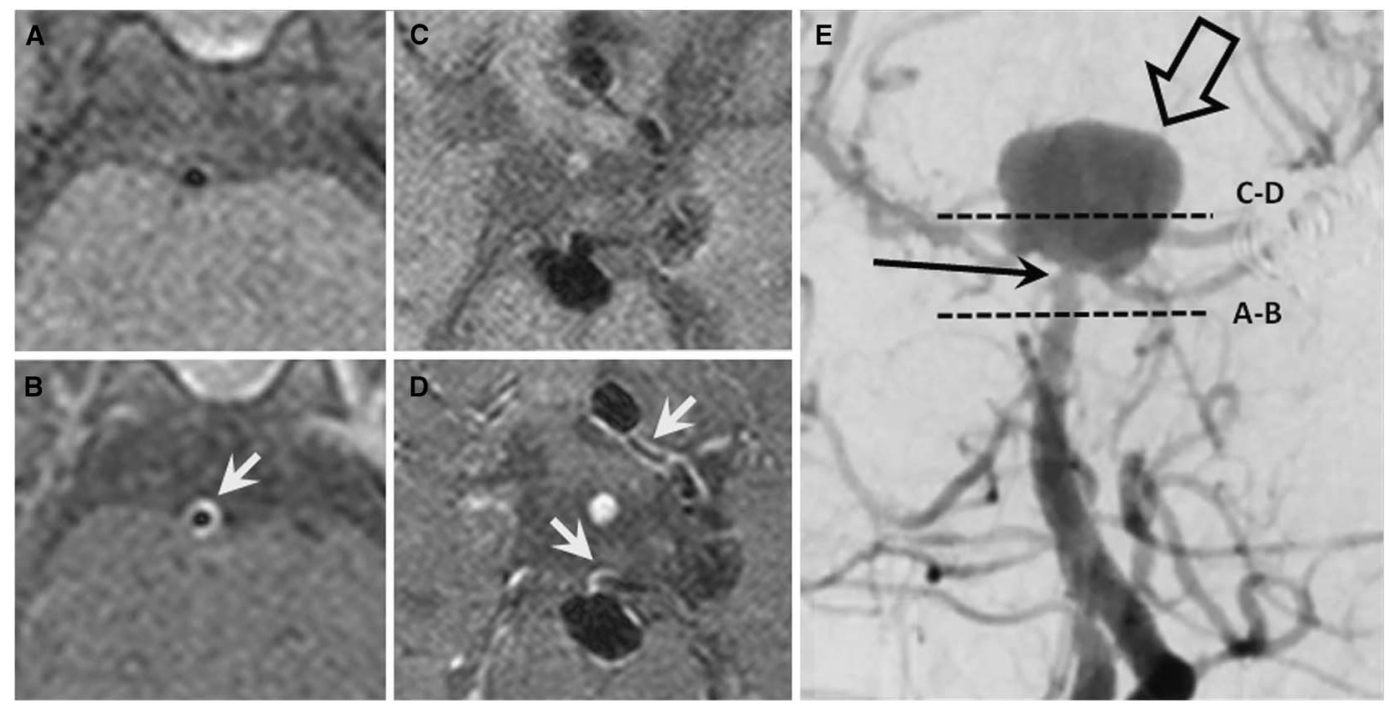

Figure 2: MRI axial TISE-weighted sequence (level of slices represented as a dotted line in E) performed before $(A \& C)$ and after gadolinium injection $(B \& D)$ and angiography (E: basilar artery) in frontal view, both performed in December 2011. Persistence of diffuse vessel wall enhancement is demonstrated (B \& D: white arrows). Interval development of a similar pattern in the posterior circulation as in the carotid circulation with development of a basilar stenosis (E: arrow) and basilar tip aneurysm ( $D$ and E: empty arrow).

system. In such cases, we think that short-interval follow-up vessel wall MRI should be performed to adapt further treatment strategies. In our case, we decided to prophylactically treat the patient with transcortical reperfusion in order to improve vascular reserve in case of increasing posterior circulation stenosis.

Vessel wall enhancement on MRI is a known diagnostic sign of cerebral vasculitis. ${ }^{2,4}$ In our case, vessel wall enhancement involved large-sized cerebral arteries in the anterior and posterior circulation, with both arterial stenosis and aneurysmal dilatation.

Cerebral arteriopathy, which on luminal imaging appears as arterial stenosis, is common in children with acute ischaemic stroke. Associated risk factors are early school age, sickle cell disease and recent upper respiratory infections. ${ }^{1}$ In our presentation, the patient did not have any of these risk factors. Moreover, no data are available in the literature regarding arterial dilatations or aneurysms. Although the patient had a presumed CNS vasculitis and had elevated anticardiolipin antibodies, he did not fit the classical presentation of focal cerebral arteriopathy of childhood. ${ }^{1,3}$

To conclude, we observed that wall enhancement on 2-mm T1SE-weighted sequences in case of cerebral vasculitis was followed by further changes in arteries that appear initially normal on angiography in a patient who was a poor responder to medical treatment. Further studies are needed to explore this relationship 
and to determine if vessel wall enhancement could predict further appearance of stenosis and/or aneurysmal dilatations in such cases.

\section{DisClOSURES}

The authors hereby declare that they have no interests that might be perceived as posing a conflict or bias.

\section{CONFLicts OF INTEREST}

The authors state that they have no conflicts of interest to declare.

\section{REFERENCES}

1. Amlie-Lefond C, Bernard TJ, Sébire G, Friedman NR, Heyer GL, Lerner NB, et al. Predictors of cerebral arteriopathy in children with arterial ischemic stroke: results of the International Pediatric Stroke Study. Circulation. 2009;119(10):1417-23.

2. Küker W, Gaertner S, Nagele T, Dopfer C, Schoning M, Fiehler J, et al. Vessel wall contrast enhancement: a diagnostic sign of cerebral vasculitis. Cerebrovasc Dis. 2008;26(1):23-9.

3. Mineyko A, Narendran A, Fritzler ML, Wei XC, Schmeling H, Kirton A. Inflammatory biomarkers of pediatric focal cerebral arteriopathy. Neurology. 2012;79(13):1406-8.

4. Saam T, Habs M, Pollatos O, Cyran C, Pfefferkorn T, Dichgans M, et al. High-resolution black-blood contrast-enhanced T1-weighted images for the diagnosis and follow-up of intracranial arteritis. Br J Radiol. 2010;83(993):e182-4. 